\title{
APRENDIZAGEM DE RELAÇÕES PALAVRA-OBJETO POR BEBÊS EM UM PROCEDIMENTO DE INTRODUÇÃO SUCESSIVA DE ESTÍMULOS
}

\author{
LEARNING OF WORD-OBJECT RELATIONS IN INFANTS IN A SUCCESSIVE INTRODUCTION OF STIMULUS \\ PROCEDURE
}

JULIA RIBEIRO

ANDRÉIA SCHMIDT

UNIVERSIDADE DE SÃo PAULO, BRASIL E

InSTITUTO NACIONAL DE CiênCIA E TECNOLOGIA SOBRE COMPORTAMENTO, CoGNiÇão E ENSINO, BRASIL

\begin{abstract}
RESUMO
O desempenho por exclusão, definido como a seleção de um estímulo indefinido (entre alternativas conhecidas), diante de um modelo também indefinido, é referido como um componente importante na aprendizagem de vocabulário em crianças pequenas. Apesar de ser um fenômeno bem documentado pela literatura, é necessário ainda compreender como a apresentação de novas palavras para bebês pode afetar a aprendizagem de múltiplas relações nome-objeto. O objetivo deste estudo foi verificar se o procedimento de introdução sucessiva de estímulos é eficiente para ensinar simultaneamente duas relações nome-objeto para bebês. Participaram sete bebês (três meninas), de 17 a 20 meses de idade. Foram realizadas tarefas de emparelhamento ao modelo com objetos e nomes conhecidos (linha de base), seguidas de blocos de ensino de duas novas relações nome-objeto, com a introdução sucessiva das novas relações, e testes de aprendizagem. Todas as crianças responderam por exclusão na primeira apresentação de cada estímulo novo, confirmando a ocorrência desse desempenho em bebês. A introdução da segunda nova relação desestabilizou o desempenho dos participantes no bloco de ensino, mas dois dos sete bebês mostraram indícios de aprendizagem das relações nos testes. A condução de testes em extinção pode ter gerado um efeito deletério no desempenho dos bebês. A introdução sucessiva de novas relações nome-objeto pode ser uma forma viável de ensino de novas relações para crianças pequenas, mas deve-se ainda investigar o número necessário de exposições dessas relações e reavaliar a pertinência de testes em extinção para bebês, uma vez que seu efeito pode mascarar a aprendizagem desses participantes.

Palavras-chave: responder por exclusão, aprendizagem de vocabulário, procedimento de introdução sucessiva de estímulos, bebês.

Exclusion performance is defined as the selection of an undefined stimulus (amongst known alternatives), when an undefined sample is presented. This process is referred to as an important component in vocabulary learning in young children. Despite being a well-documented phenomenon in literature, it is necessary to understand how the presentation of new words to infants can affect learning of multiple word-object relations. The aim of this study was to determine whether the successive introduction of stimuli procedure is efficient to teach simultaneously two new word-object relations for infants. Participants were seven children (17-20 months old - three girls). Matching to sample tasks were carried out with familiar objects and names (baseline), followed by one block of training trials (reinforced exclusion trials) of two new word-object relations (successive introduction of stimuli procedure), and learning tests. All children presented exclusion performance in the first presentation of each new stimulus, confirming the occurrence of this performance in infants. The introduction of the second new relation disturbed the participants' performance in the teaching trials, but two out of seven children showed evidence of learning in probes. Tests in extinction may have generated a deleterious effect on the infants' performance. The successive introduction of new word-object relations can be a viable way of teaching words for young children, but it is necessary to investigate the necessary number of teaching trials and to evaluate the effects of tests in extinction for infants, since its effect can mask the learning of these participants.
\end{abstract}

Keywords: responding by exclusion, vocabulary learning, successive introduction of stimuli procedure, infants.

Andréia Schmidt é docente da Faculdade de Filosofia, Ciências e Letras de Ribeirão Preto (USP - FFCLRP), Departamento de Psicologia. Av. Bandeirantes, 3900, Bairro Monte Alegre, Ribeirão Preto, São Paulo, Brasil. CEP: 14040-901. Tel.: (16) 3315-3742. E-mail: aschmidt@,ffclrp.usp.br. É também membro do Instituto Nacional de Ciência e Tecnologia sobre Comportamento, Cognição e Ensino (INCT-ECCE), financiada pelo Conselho Nacional de Pesquisa (CNPq, Processo no 573972/2008-7) e pela Fundação de Amparo à Pesquisa do Estado de São Paulo (FAPESP, Processo $n^{\circ} 08 / 57705-8$ ). 
A aprendizagem de comportamento verbal é uma das principais conquistas culturais dos primeiros anos da vida de uma criança. A aprendizagem desse repertório sofisticado parece ocorrer de forma natural, sem a necessidade de ensino direto e específico. No entanto, incontáveis interações entre a criança e membros de sua comunidade verbal ocorrem diariamente e são os fatores cruciais no desenvolvimento dos repertórios verbais das crianças (Greer \& Ross, 2008). Nessas interações uma série de processos de aprendizagem se entrelaçam para compor o vasto repertório que crianças ainda pequenas apresentam (Kucker, McMurray, \& Samuelson, 2015) e o estudo desses processos é crucial para a compreensão da complexidade das interações verbais entre os indivíduos.

Um dos processos que tem interessado analistas do comportamento e psicolinguistas é o responder por exclusão, termo cunhado por Dixon (1977) e que faz parte de um processo mais amplo denominado na Psicolinguística de fast mapping (Carey \& Bartllet, 1978). $\mathrm{O}$ interesse por esse processo vem do fato de que, aproximadamente a partir dos 18 meses, as crianças passam a aprender muitas novas relações entre palavras e referentes (e.g., objetos, figuras, ações, propriedades de objetos e ações, relações entre objetos), de forma acelerada e sem que seja possível identificar episódios de instrução específica ou de reforçamento diferencial para todas essas aprendizagens. Ao menos aparentemente, o desempenho por exclusão poderia ser um fator central para a aprendizagem dessas novas relações, uma vez que ele consiste no estabelecimento de uma relação imediata entre um estímulo modelo indefinido (e.g., uma palavra desconhecida dita por um adulto) e um estímulo de comparação também indefinido (e.g., um objeto desconhecido presente na situação e que está entre objetos familiares), sem uma história prévia que torne o estímulo de comparação discriminativo para sua escolha diante daquele modelo (McIlvane \& Stoddard, 1981). Neste contexto, uma criança poderia aprender a relação entre um brinquedo que ela nunca viu anteriormente e um nome ouvido pela primeira vez, em uma situação em que essa palavra é dita na presença do brinquedo novo e de outros que ela já conhece. Os brinquedos familiares, nesse caso, funcionariam como "contraste negativo" ( $\left.\mathrm{S}^{-}\right)$para o estabelecimento imediato de uma relação entre os estímulos visual e auditivo desconhecidos.

Esse fenômeno tem sido extensamente pesquisado e demonstrado na literatura, em indivíduos de diferentes idades (e.g., Costa, McIlvane, Wilkinson, \& de Souza, 2001; Domeniconi, Costa, de Rose, \& de Souza, 2007; Wilkinson \& McIlvane, 1997) e condições de desenvolvimento (e.g., Carr, 2003; Greer \& Du, 2015; Langsdorff, Domeniconi, Schmidt, de Souza, \& Gomes, in press), o que indica que o responder por exclusão é um desempenho altamente provável, mesmo em crianças préverbais (Mather \& Plunkett, 2011). Grande parte das pesquisas sobre esse tema, porém, é desenhada para investigar a aprendizagem de uma única relação nomeobjeto por vez (e.g., Domeniconi et al., 2007; Horst \& Samuelson, 2008; Langsdorff, Schmidt, \& Domeniconi, 2015), o que pode ser importante para a investigação mais apurada de variáveis específicas envolvidas no processo (como o número necessário de exposições à palavra para a aprendizagem da relação testada, por exemplo), mas que não replica condições reais às quais os bebês estão expostos no seu cotidiano. É muito comum, especialmente no caso de bebês mais jovens, que os adultos pronunciem mais de uma palavra desconhecida na fala dirigida ao bebê, em ambientes com mais de um objeto desconhecido. Nesse caso, a criança seria capaz de aprender de forma rápida (i.e., a partir de um número pequeno de exposições) duas ou mais novas relações nome-objeto ao mesmo tempo? O responder por exclusão poderia favorecer essa aprendizagem?

O estudo dessa questão com adultos mostra que eles são capazes de aprender de forma fácil e rápida relações múltiplas entre palavras e objetos desconhecidos (e.g., Golinkoff, Hirsch-Pasek, Bailey, \& Wenger, 1992 Experimento 1). Estudar a aprendizagem de múltiplas relações nome-objeto com bebês, porém, demanda a programação de condições experimentais que favoreçam o seu engajamento à tarefa, além de evidenciar o efeito das variáveis estudadas (Oliveira \& Gil, 2008). Em um estudo com crianças na faixa dos 2 anos e meio Golinkoff et al. (1992 - Experimento 2) verificaram que os participantes demonstraram aprendizagem de duas novas relações nome-objeto a partir de um número bastante reduzido de exposições a situações em que o responder por exclusão era favorecido. No entanto, o estudo foi realizado com apenas duas exposições a cada palavra e com poucas tentativas de teste de aprendizagem, o que pode ter enviesado os resultados, especialmente porque há estudos que mostram que crianças nessa faixa etária, em geral, não aprendem novas relações entre palavras e objetos a partir de um número tão reduzido de tentativas de exclusão (e.g., Domeniconi et al., 2007; Schmidt, Franco, Lotério, \& Gomes, 2016).

Estudos com crianças de 15 e 16 meses atestam a dificuldade de aprendizagem de duas relações nomeobjeto ao mesmo tempo nessa faixa etária. Schafer e Plunkett (1998), por exemplo, apresentaram duas relações nome-figura para bebês de 15 meses em dois blocos de 12 tentativas e conduziram testes imediatamente após essas exposições. Os autores encontraram evidências de que os bebês aprenderam pelo menos uma das novas relações, mas poucos mostraram aprendizagem das duas. Liittschwager e Markman (1994), estudando bebês de 16 meses, verificaram que a grande maioria dos seus participantes foram capazes de aprender uma nova relação nome-objeto, mas não conseguiram aprender um segundo nome para um objeto já conhecido. Em ambos os estudos, a forma de apresentação das relações nome-figura/objeto era o pareamento entre os estímulos, e não procedimentos que favorecessem a ocorrência do responder por exclusão.

Wilkinson e Green (1998) estudaram a aprendizagem de duas novas relações nome-figura com indivíduos com deficiência intelectual, cuja idade de vocabulário estava entre 2 e 6 anos. Para isso, desenvolveram duas estratégias de apresentação das novas relações. Em uma delas, chamada de introdução concorrente de estímulos, as duas novas relações seriam 
ensinadas em duas sessões de ensino e outra de testes: na primeira sessão, uma nova palavra seria apresentada ao participante diante de um conjunto de duas figuras conhecidas e apenas uma desconhecida (seis exposições); na segunda sessão, uma segunda palavra nova seria apresentada diante de duas figuras conhecidas e uma desconhecida (seis exposições); finalmente, na sessão de testes, as duas novas palavras seriam apresentadas no mesmo bloco de tentativas e os comparações seriam sempre uma figura conhecida e as duas figuras ensinadas nas sessões anteriores. $\mathrm{Na}$ condição de introdução sucessiva, na primeira sessão uma nova palavra era apresentada diante de duas figuras familiares e uma desconhecida (seis exposições); na segunda sessão, a segunda relação era introduzida, mas os comparações eram uma figura familiar, uma figura completamente nova e a figura relacionada à palavra desconhecida na sessão anterior, que deveria funcionar como um estímulo definido para a nova relação, tal como a figura familiar. $\mathrm{Na}$ sessão de testes as duas palavras eram apresentadas de forma não consecutiva na sessão, e os comparações eram uma figura familiar e as duas ensinadas. A configuração das tentativas de teste era, portanto, idêntica à da segunda sessão de ensino.

Wilkinson e Green (1998) verificaram que a exposição sucessiva foi mais eficiente no ensino das duas relações para os participantes com deficiência intelectual do que a condição de exposição concorrente. Empregando o procedimento de introdução sucessiva, Carr (2003) ensinou de forma bem-sucedida quatro novas relações nome-objeto a crianças com autismo. Mas, e crianças com desenvolvimento típico apresentariam diferentes desempenhos nas duas condições?

Wilkinson, Ross e Diamond (2003), aplicaram os procedimentos de introdução concorrente e introdução sucessiva de estímulos a 58 crianças de 26 a 57 meses de idade, no ensino de duas novas relações nome-objeto. As crianças mais novas demonstraram melhor aprendizagem das novas relações pelo procedimento de introdução sucessiva, provavelmente, segundo as autoras, porque as tentativas de ensino conduzidas na apresentação da segunda relação eram semelhantes às tentativas de teste, promovendo a discriminação dos dois objetos "novos". No entanto, é preciso considerar que as crianças mais novas do estudo de Wilkinson et al. (2003) tinham 26 meses.

O estudo de Costa, Grisante, Domeniconi, de Rose e de Souza (2013), realizado com crianças entre 4 e 5 anos, também investigou a aprendizagem de duas novas relações nome-figura em um procedimento de ensino por exclusão modificado, incluindo uma medida de nomeação dos estímulos e controlando o número de exposições necessárias até que as crianças nomeassem as novas figuras. Após uma linha de base com figuras e palavras conhecidas, as autoras apresentavam às crianças duas tentativas de exclusão, distribuídas entre algumas tentativas de linha de base, cada uma apresentando uma nova palavra diante de uma figura conhecida, uma desconhecida e um comparação vazio (ver Wilkinson \& McIlvane, 1997, para a discussão da função do comparação vazio). Na sequência, a criança era exposta a sondas de nomeação (para verificar se ela era capaz de dizer o nome das figuras novas) e quatro sondas de aprendizagem: para cada relação eram apresentadas duas sondas, uma em que a palavra nova era ditada e os comparações eram as duas novas figuras e um comparação vazio, e outra que apresentava uma figura conhecida, uma completamente nova e o comparação vazio. Todas as crianças demonstraram aprendizagem das duas relações, inclusive com a nomeação correta dos dois estímulos visuais, mas após um número variável de exposições às tentativas de exclusão (entre três e dez exposições). Nesse estudo, a forma de apresentação das novas relações não previa o ensino direto de cada uma delas; verificava-se, apenas, se a simples exposição a tentativas de exclusão não reforçadas seria suficiente para promover a aprendizagem dessas relações, o que foi aferido em tarefas de nomeação e de emparelhamento ao modelo.

Os resultados dos estudos aqui descritos indicam que o desempenho por exclusão pode ser importante na aprendizagem de múltiplas relações, especialmente em crianças com mais de dois anos. Entretanto, é importante verificar se o mesmo ocorre em crianças menores, com repertório verbal ainda pouco desenvolvido, uma vez que essa informação pode auxiliar na compreensão de processos envolvidos na aprendizagem de relações múltiplas em bebês com repertório verbal incipiente. Esse estudo teve como objetivo verificar se o procedimento de introdução sucessiva de estímulos é eficiente para o ensino de duas novas relações palavra-objeto para bebês com idade entre 17 e 20 meses.

Esse procedimento foi escolhido em função de resultados positivos encontrados na sua aplicação em indivíduos com problemas de desenvolvimento e repertório verbal pouco desenvolvido (Carr, 2003; Wilkinson \& Green, 1998), além de ter sido indicado por Wilkinson et al. (2003) como mais eficiente para crianças com desenvolvimento típico mais jovens. O número de exposições na fase de ensino foi determinado a partir do número de tentativas descritos em outros estudos com crianças: Schwartz e Terrell (1983) afirmam que crianças entre 12 e 18 meses levaram, em média, de 10 a 12 exposições a uma palavra nova para serem capazes de produzi-la adequadamente; o estudo de Costa et al. (2013) evidenciou que crianças de quatro anos podem aprender duas novas relações a partir de um número de exposições que variou entre duas e 10; por fim, Wilkinson et al. (2003) trabalharam com três exposições a cada uma das novas relações ensinadas. Os parâmetros adotados no presente estudo serão melhor descritos no Método.

\section{MÉTODO}

\section{Participantes}

Participaram dessa pesquisa sete bebês (três meninas) com idade entre 17 e 20 meses (média de 19,1 meses), que frequentavam creches, todos sem indícios de atraso do desenvolvimento de acordo com relato de professores e pais. Todos os pais autorizaram a participação das crianças, assinando um Termo de Consentimento Livre e Esclarecido antes do início do procedimento. As sessões foram realizadas em uma sala 
da escola onde as crianças estudavam, sem a presença de outras crianças ou de adultos, além das pesquisadoras.

\section{Materiais e equipamentos}

Foram utilizados quatro brinquedos conhecidos pelas crianças (boneca, carrinho, bola e cachorro de pelúcia) e dois brinquedos não familiares, sem forma definida e que não estavam relacionados a qualquer nome já conhecido pela criança. A familiaridade dos bebês com os objetos foi verificada, inicialmente, junto à professora, mas posteriormente confirmada na linha de base (ver Procedimentos). As sessões foram registradas com uma filmadora digital.

\section{Procedimentos}

A pesquisa foi conduzida de acordo com a legislação brasileira sobre ética em pesquisas com seres humanos e aprovado pelo Comitê de Ética em Pesquisa da FFCLRP-USP (CAAE $n^{\circ}$ 11619913.7.0000.5407). Após duas semanas de adaptação, nas quais a pesquisadora frequentou a classe das crianças e permaneceu com elas por pelo menos uma hora por dia, foi dado início ao procedimento. Individualmente, as crianças eram levadas para uma sala e convidadas para uma brincadeira. A situação experimental geral foi baseada no estudo de Wilkinson et al. (2003), com algumas modificações em relação ao número de tentativas de treino, às palavras e aos objetos utilizados. A pesquisadora, uma auxiliar de pesquisa e a criança ficavam sentadas no chão. A auxiliar ficava atrás da criança (às vezes com ela no colo) e, em frente à criança, eram colocados os brinquedos, lado a lado. A pesquisadora ficava de frente para a criança, olhando em direção a ela enquanto dava as instruções ou ditava o nome do brinquedo a ser selecionado. Esse cuidado foi tomado para que a direção do olhar da pesquisadora não servisse de dica para a criança sobre a resposta correta. Todas as tentativas eram de matching-to-sample (MTS), nas quais, após a pesquisadora ditar uma palavra, a criança deveria localizar o brinquedo ditado, pegá-lo e entregá-lo para a pesquisadora. O tempo total do procedimento com cada criança foi de, no máximo, 10 minutos. Inicialmente, foi estabelecida uma linha de base com brinquedos conhecidos, seguida da fase de ensino das novas relações palavra-objeto e das sondas de aprendizagem, conforme descrição a seguir (ver Figura 1).

Linha de base. $O$ objetivo dessa fase foi estabelecer a resposta de selecionar um brinquedo condicionalmente ao nome falado pela pesquisadora. Foram realizadas cinco tentativas de MTS auditivo-visual. Em cada tentativa, a pesquisadora ditava o nome de um dos três brinquedos conhecidos dispostos à frente da criança: "Fulano, qual desses brinquedos é [a bola]? Pega pra mim [a bola]". Em seguida, era esperado que a criança emitisse qualquer resposta em direção ao objeto solicitado (tocar, pegar, entregar ou apontar). Assim que a criança emitisse essa resposta, a pesquisadora e a auxiliar elogiavam a criança e batiam palmas, consequenciando, assim, o seu desempenho. Se a criança pegasse o objeto incorreto, a pesquisadora dizia "Não, não é esse. Vamos tentar de novo? Onde está a bola?". Essa fase era encerrada após cinco tentativas corretas sucessivas.

\section{Linha de Base}

Qual desses brinquedos é a bola?

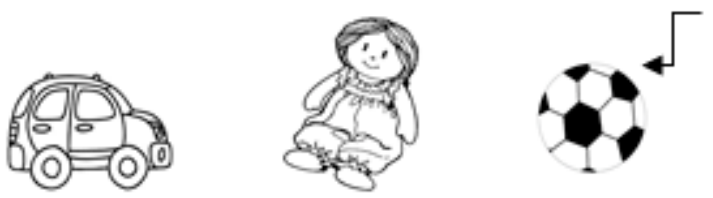

Ensino

Qual desses brinquedos é o "Fulito"?

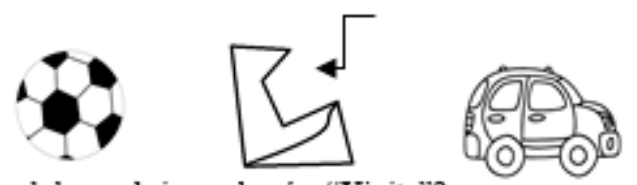

Qual desses brinquedos é o "Xipite"?

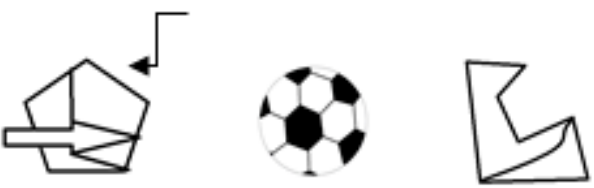

Teste

Qual desses brinquedos é o "Fulito"?
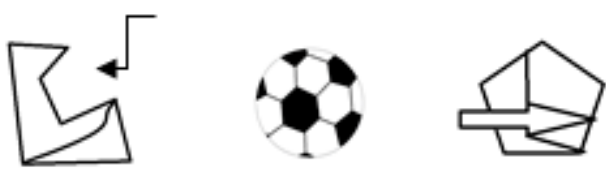

Qual desses brinquedos é o"Xipite"?
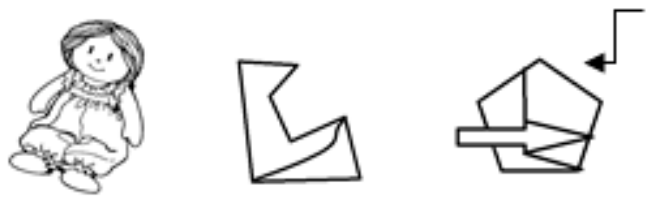

Figura 1. Exemplos esquemáticos de tentativas das diferentes fases do procedimento.

Ensino de novas relações palavra-objeto. O objetivo dessa fase era ensinar as relações entre os estímulos auditivos e visuais, consequenciando diferencialmente o desempenho dos participantes nas tentativas de exclusão (a rigor, considera-se que o desempenho por exclusão ocorre apenas na primeira apresentação do modelo indefinido diante do comparação também desconhecido; para facilitar a descrição, no entanto, serão chamadas de "tentativas de exclusão" todas as tentativas envolvendo os modelos indefinidos, mesmo aquelas consequenciadas diferencialmente). Na sequência da linha de base, uma palavra desconhecida ("Fulito") era ditada, em cinco tentativas de ensino não consecutivas (alternadas com tentativas de linha de base). Em cada tentativa de ensino a palavra desconhecida era apresentada três vezes: "Pega o Fulito para mim? Qual desses é o Fulito? Me dá o Fulito?", e como comparações eram apresentados dois brinquedos conhecidos e um desconhecido (D1). Em todas as tentativas, a posição dos brinquedos era alternada, assim como a posição do brinquedo correto. No total, a nova palavra era apresentada por 15 vezes (cinco tentativas, com três vezes 
a palavra ditada em cada uma). Na sequência, a segunda palavra desconhecida ("Xipite") era apresentada, também em cinco tentativas alternadas com tentativas de linha de base. Nessas tentativas de ensino, estavam disponíveis para escolha os dois objetos desconhecidos (D1 e D2), junto a um brinquedo familiar que se alternava entre as tentativas. Tanto nas tentativas de linha de base, quanto nas tentativas de ensino, escolhas corretas eram consequenciadas com aplausos e elogios; tentativas incorretas eram corrigidas ("Não, não é esse, vamos tentar de novo?"). Finalizadas as tentativas de ensino das duas novas palavras, eram apresentadas as sondas de aprendizagem.

Sondas de aprendizagem. Nessa fase, o objetivo era verificar se as relações ensinadas na fase anterior se manteriam em tentativas em que os dois estímulos novos eram apresentados juntos, alternando-se a apresentação dos modelos. As Sondas de Aprendizagem eram apresentadas em um bloco de oito tentativas, três para cada palavra ensinada, entre as quais eram apresentadas duas tentativas de linha de base. Nessas tentativas, tanto D1 como D2 estavam disponíveis para escolha, juntos com um objeto familiar. A posição dos comparações mudava a cada tentativa. Nenhuma tentativa (de sonda ou de linha de base) era consequenciada diferencialmente; qualquer que fosse a resposta da criança, passava-se para a tentativa seguinte (e.g., "Agora, mostre para mim onde está o Fulito."). O procedimento se encerrava após a última tentativa do bloco.

Todo o procedimento foi registrado em vídeo para posterior registro das respostas dos participantes. Um segundo observador assistiu a $30 \%$ dos vídeos das sessões e fez o registro de acertos e erros dos participantes. O cálculo de concordância foi feito dividindo-se o número de acordos pela soma de acordos e desacordos, multiplicado por 100 (Danna \& Matos, 2006). O índice de concordância entre os observadores foi de $100 \%$.

\section{RESULTADOS}

Todos os bebês atingiram o critério de acertos na Linha de Base sem qualquer erro, indicando que eles conheciam os brinquedos apresentados e se familiarizaram rapidamente à tarefa. Os desempenhos individuais das crianças no Ensino de Novas Relações Palavra-Objeto para as duas palavras são apresentados na Figura 2.

Observa-se que, no ensino da primeira palavra (Fulito) todos os bebês apresentaram desempenho por exclusão na primeira tentativa, e mantiveram-se escolhendo o brinquedo D1 ao longo de todas as tentativas de ensino dessa palavra, com exceção de P5 e P6, ambos de 20 meses, que erraram uma tentativa cada. Com a introdução da segunda nova palavra (Xipite), todas as crianças novamente selecionaram o brinquedo desconhecido (D2) na primeira tentativa; no entanto, cinco das sete crianças apresentaram erros na segunda tentativa de ensino da palavra Xipite. Apenas P6 manteve-se selecionando corretamente o brinquedo D2 diante da palavra Xipite ao longo de todas as tentativas de ensino e P7 (20 meses), apresentou um único erro na quarta tentativa de ensino da segunda palavra. Todas as demais crianças, a despeito de terem respondido por exclusão na primeira tentativa de ensino de cada palavra, apresentaram pelo menos dois erros nas tentativas relacionadas à segunda nova palavra.
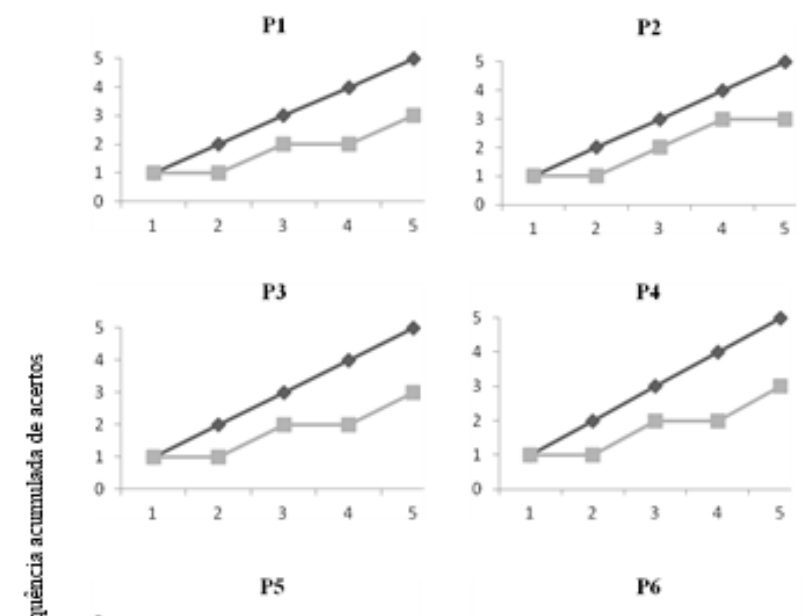

$$
\begin{array}{ll}
\text { 嵒 } & 5 \\
4 \\
3 \\
\\
2 \\
\\
1 \\
0
\end{array}
$$
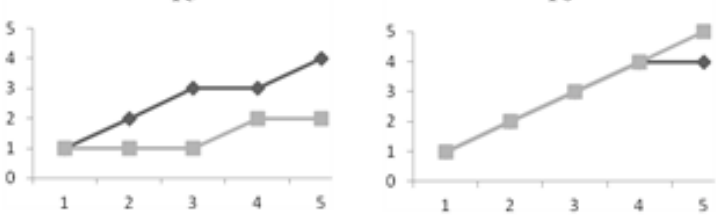

P7

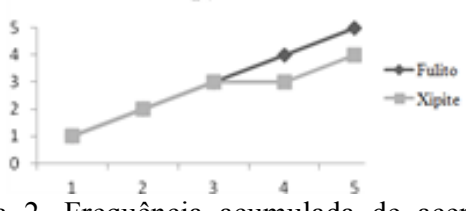

Figura 2. Frequência acumulada de acertos nas tentativas de ensino das palavras Fulito (primeira palavra ensinada) e Xipite (segunda palavra ensinada).

Os desempenhos individuais das crianças nas Sondas de Aprendizagem são apresentados na Figura 3.

Observa-se na Figura 3 que cinco (P1, P3, P4, P5 e P7), das sete crianças, acertaram a primeira tentativa de sonda apresentada. No entanto, a partir da tentativa seguinte, os resultados foram variáveis. P6 e P7 foram os participantes que apresentaram uma sequência de quatro escolhas corretas, com declínio no desempenho nas últimas tentativas de sonda do bloco. Esses dois participantes apresentaram os resultados mais consistentes e o declínio de desempenho observado lembra uma curva de extinção. P1 (17 meses) também apresentou quatro desempenhos corretos (com um erro entre o primeiro e o segundo acerto), o que sugere, também, um desempenho consistente, mas que declinou no final. P3 (19 meses) apresentou três acertos consecutivos nas três primeiras tentativas de sonda, passando, então, a selecionar apenas o brinquedo D2 em todas as tentativas. P2, P4 e P5, no entanto, apresentaram um número grande de erros ao longo de todo o bloco de sondas em relação às duas palavras, o que indica que nenhum desses três participantes aprendeu de forma consistente a relação entre os nomes ditados e os brinquedos desconhecidos. 

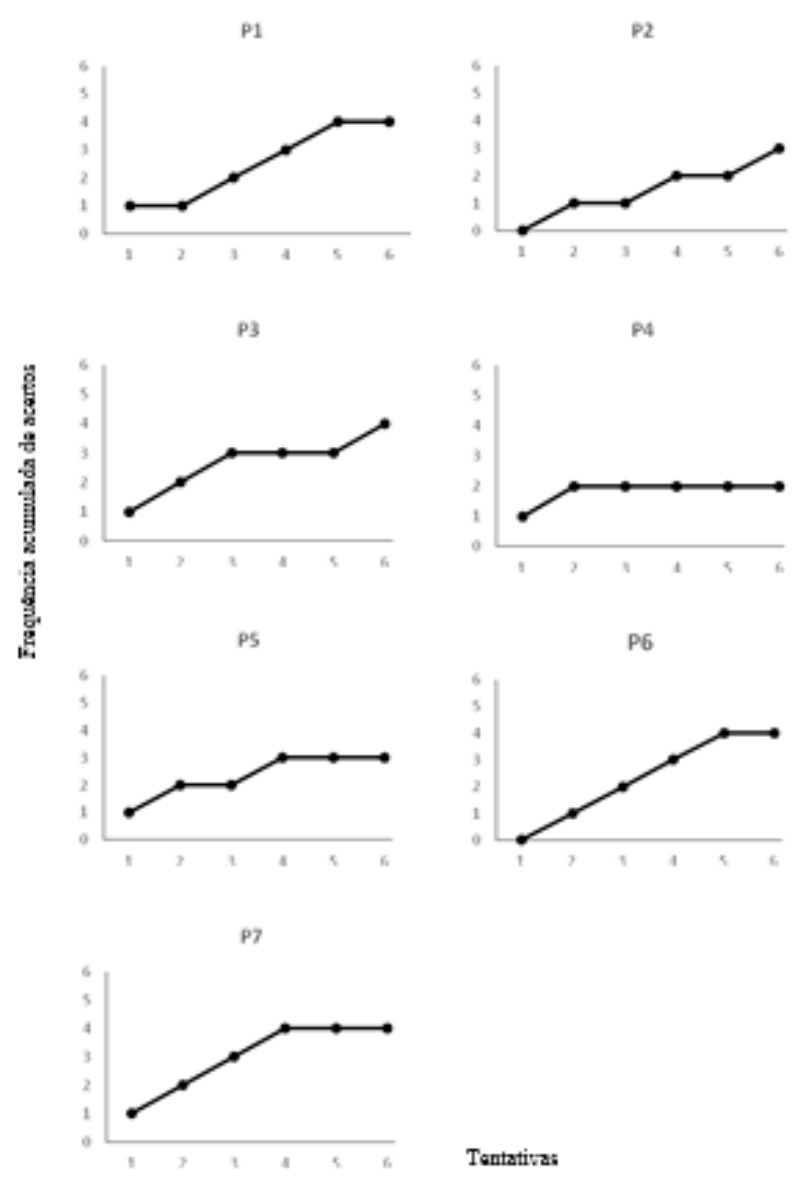

Figura 3. Frequência acumulada de acertos nas tentativas de sonda com as duas palavras novas.

A sequência de apresentação das palavras foi: Xipite, Fulito, Xipite, Fulito, Fulito e Xipite

Também é importante verificar se as crianças aprenderam de forma diferente as duas palavras, uma vez que a maioria dos participantes não apresentou erro nas tentativas de ensino da palavra Fulito, mas apresentou mais dificuldades com a introdução da segunda palavra nova (Xipite). A Tabela 1 apresenta o desempenho correto de cada participante em relação às palavras individuais.

Tabela 1

Número de Desempenhos Corretos dos Participantes Individuais para Cada Palavra nas Sondas de Aprendizagem.

\begin{tabular}{ccc}
\hline Participante & Fulito & Xipite \\
\hline P1 & 2 & 2 \\
P2 & 2 & 1 \\
P3 & 3 & 1 \\
P4 & 1 & 1 \\
P5 & 1 & 2 \\
P6 & 3 & 1 \\
P7 & 2 & 2 \\
\hline Porcentagem & $61 \%$ & $52 \%$ \\
média de acertos &
\end{tabular}

Nota: Três era o número máximo de acertos para cada palavra

Pode-se observar que os três participantes com os melhores resultados em aprendizagem (P1, P6 e P7) tiveram desempenhos diferentes em relação às duas palavras: P7 teve dois acertos em três das tentativas para cada palavra, com erros ocorrendo ao final do procedimento; P6 acertou as três tentativas com Fulito e apenas uma com Xipite, o que pode indicar preferência por um dos objetos; e P1 apresentou dois acertos para cada palavra. Os demais participantes não tiveram desempenho acurado em qualquer uma das duas palavras.

\section{DISCUSSÃO}

O objetivo da presente pesquisa foi verificar se o procedimento de introdução sucessiva de estímulos seria eficiente para ensinar duas novas relações palavra-objeto para bebês com idade entre 17 e 20 meses. De modo geral, verificou-se que todos os bebês responderam por exclusão na apresentação das duas relações novas (Fulito e Xipite), mas que apenas dois deles (P1 e P7) apresentaram indícios mais consistentes de aprendizagem dessas relações. A análise de variáveis relacionadas ao procedimento e às condições experimentais pode auxiliar na compreensão desses resultados.

O desempenho dos bebês deste estudo nas tentativas de exclusão replica resultados de outras pesquisas realizadas com crianças de diferentes idades e com diferentes níveis de desenvolvimento (e.g., Costa et al., 2001; Domeniconi et al., 2007; Horst \& Samuelson, 2008; Markman, Wasow, \& Hansen, 2003; Schmidt et al., 2016; Wilkinson \& McIlvane, 1997). Além de demonstrarem a ocorrência desse desempenho emergente em bebês com repertório verbal ainda inicial, os resultados sugerem que procedimentos de ensino por exclusão podem ser empregados com crianças nessa idade, desde que estabelecida uma linha de base consistente com estímulos familiares.

Procedimentos de ensino por exclusão têm sido desenvolvidos e testados em muitos estudos, inclusive com crianças e indivíduos com deficiência intelectual (e.g., de Souza \& de Rose, 2006; Dixon, 1977; Ferrari, de Rose, \& McIlvane, 1993; McIlvane \& Stoddard, 1981; Wilkinson, Rosenquist, \& McIlvane, 2009). O presente estudo utilizou um procedimento de ensino por exclusão denominado de introdução sucessiva de estímulos. Esse tipo de procedimento, com algumas variações, já foi utilizado de forma bem-sucedida em outros estudos com crianças mais velhas (e.g., Carr, 2003; Wilkinson et al., 2003). Nesse procedimento as duas relações são ensinadas quase simultaneamente, o que replica a situação de teste de aprendizagem e pode servir como uma situação de ensino da tarefa que será proposta à criança nas sondas.

No estudo de Wilkinson et al., (2003), assim como no presente estudo, a introdução da segunda nova palavra como modelo na presença dos dois objetos desconhecidos, desestabilizou o desempenho de parte dos participantes: cerca de $40 \%$ das crianças com menos de 42 meses que passaram por esse procedimento no estudo de Wilkinson et al. acertaram apenas uma das três tentativas de ensino conduzidas; no presente estudo, cinco das sete crianças apresentaram dois ou três erros ao longo das cinco tentativas de ensino (as exceções foram P6 e P7, que apresentaram um único erro). A ocorrência de erros na introdução da segunda relação era, portanto, esperada. A presença de dois estímulos com os quais a criança tinha 
pouca ou nenhuma experiência anterior poderia tornar mais difícil o estabelecimento das duas relações palavraobjeto, ainda que, anteriormente, os bebês tivessem passado por um bloco de tentativas reforçadas para a escolha do primeiro objeto novo diante da palavra Fulito. Por outro lado, também era esperado que, a despeito da ocorrência desses erros, a sequência de tentativas de ensino da segunda nova relação pudesse favorecer a discriminação das características dos objetos relacionados a diferentes palavras. $\mathrm{O}$ desempenho das cinco crianças que apresentaram dois ou mais erros no ensino da segunda relação pode dar alguns indícios sobre se, de fato, a presença dos dois estímulos pouco conhecidos auxiliou essa discriminação ao longo do ensino.

Todas as crianças responderam por exclusão na primeira apresentação da segunda palavra nova, mas erraram a segunda tentativa de ensino da segunda relação, tendo os dois objetos pouco conhecidos como alternativa de escolha. $\mathrm{O}$ fato de terem errado a segunda tentativa pode sugerir que as crianças estavam sob controle da dimensão de novidade do segundo estímulo quando este foi apresentado pela primeira vez, e não sob controle de características específicas do brinquedo; dessa forma, após terem selecionado o objeto novo na tentativa de exclusão, na segunda apresentação da palavra desconhecida e diante dos dois objetos pouco conhecidos, voltaram a escolher o objeto cuja seleção foi reforçada em tentativas anteriores. Ao terem o erro consequenciado, no entanto, quatro das cinco crianças selecionaram o estímulo correto diante da palavra Xipite na terceira tentativa (com exceção de P5), mas três deles voltaram a errar na quarta tentativa de ensino (P1, P3 e P4), o que gerou um número excessivo de erros no ensino da segunda relação.

Wilkinson et al. (2003) relataram que, apesar da desestabilização do desempenho de parte das crianças mais jovens do seu estudo na introdução da segunda nova relação, $60 \%$ delas atingiram $100 \%$ de respostas corretas nas sondas subsequentes das duas relações ensinadas (duas sondas por relação). Além disso, Golinkoff et al. (1992 Experimento 2), em estudo com crianças de 30 meses, verificaram indícios de discriminação de duas relações palavra-objeto a partir de um número mínimo de apresentações dos estímulos por mais da metade da amostra estudada. No presente estudo, porém, além dos bebês estudados serem bem mais jovens que os dos estudos anteriores, apenas duas, das sete crianças participantes, apresentaram um desempenho que sugere aprendizagem das duas relações ensinadas. Esses resultados chamam a atenção para a necessidade de se analisar algumas variáveis de procedimento que podem ter interferido de forma negativa no desempenho dos bebês no procedimento de ensino. Os principais pontos a serem discutidos são: a) a estabilidade do desempenho por exclusão em crianças tão jovens e os tipos de tentativas de exclusão; b) o número de tentativas de ensino conduzidas; e c) o procedimento de teste em extinção.

O desempenho por exclusão era a condição necessária para que o procedimento de ensino programado pudesse surtir resultados. Todos os participantes do presente estudo, a despeito da idade (menos de 20 meses), responderam por exclusão, tanto na apresentação do primeiro modelo indefinido, quanto na apresentação do segundo, tal como em outros estudos (e.g., Halberda, 2003 - 17 meses; Mather \& Plunkett, 2011 - 16 meses; Schmidt et al., 2016 - a partir dos 18 meses). No entanto, há que se considerar as condições sob as quais o desempenho por exclusão das crianças ocorreu nesses dois momentos.

$\mathrm{Na}$ apresentação do primeiro modelo indefinido, a criança tinha diante de si apenas um objeto desconhecido (D1), o que se constitui em uma tentativa "clássica" de exclusão (e.g., McIlvane \& Stoddard, 1981; Wilkinson \& McIlvane, 1997); a tarefa da criança era discriminar os objetos familiares do único não familiar. Na apresentação do segundo modelo indefinido, porém, havia um objeto completamente novo (D2) e outro possivelmente ainda pouco conhecido (D1). A introdução do segundo nome novo, ainda que diante de dois objetos com os quais as crianças tinham pouco ou nenhum contato anterior não perturbou a ocorrência desse desempenho, tal como ocorreu em outras pesquisas com bebês muito jovens (e.g., Liittschwager \& Markman, 1994; Schafer \& Plunkett, 1998). No entanto, a manutenção da seleção de D2 diante do modelo novo ao longo de todas as tentativas não ocorreu como com a primeira relação ensinada: a perturbação do desempenho ficou evidente ao longo das tentativas de ensino para a maioria dos participantes. Nesse caso, a criança deveria discriminar entre os dois comparações "desconhecidos", o que parece não ter ocorrido, possivelmente em função das características do procedimento adotado e dos requisitos necessários para a aprendizagem de relações verdadeiramente condicionais em uma tarefa de emparelhamento ao modelo.

$\mathrm{Na}$ proposta aqui apresentada, optou-se por um bloco de ensino com cinco tentativas em sequência para cada relação nova e com um total de 15 apresentações de cada estímulo modelo distribuídas ao longo das 10 tentativas. Esse procedimento pode ser comparável ao procedimento blocado de ensino de discriminações condicionais (Saunders \& Spradlin, 1989; 1990) também empregado no ensino de discriminações simples para crianças (Postalli, Canovas, \& de Souza (2015). Nas apresentações originais desse tipo de procedimento (e.g., Saunders \& Spradlin, 1989), os estímulos-modelo são apresentados de forma sequencial (blocos), com a redução gradual do tamanho dos blocos até que os modelos se alternem ao longo do mesmo bloco. De acordo com Saunders e Spradlin (1990), o procedimento de ensino blocado ajudaria a estabelecer uma boa discriminação, tanto entre os estímulos-modelo, como entre os estímulos de comparação. Porém, o procedimento adotado no presente estudo não previa a redução gradual das sequências de tentativas com o mesmo estímulo-modelo, o que se mostrou a grande vantagem do procedimento blocado em outros estudos. A alternância de modelos no presente estudo era realizada somente no bloco de testes e o baixo desempenho dos bebês nesses testes pode ter ocorrido exatamente porque a apresentação única de duas sequências de cinco tentativas para cada estímulo não forneceu as condições necessárias para as discriminações entre modelos e entre comparações. 
Além disso, o modelo blocado adotado também não favorecia o estabelecimento de controle do modelo sobre a seleção do comparação, o que seria uma terceira condição necessária para o estabelecimento de uma discriminação condicional (Saunders \& Spradlin, 1989). A condicionalidade entre estímulos modelo e comparações advém da alternância de funções entre os estímulos de comparação: ora eles assumem a função de $\mathrm{S}^{+}$, ora de $\mathrm{S}^{-}$, de acordo com o modelo apresentado (Dube \& McIlvane, 1992). Isso ocorreu apenas para a relação Fulito-D1, mas não para a relação Xipite-D2 uma vez que, no Ensino, D2 nunca assumiu a função de $\mathrm{S}^{-}$diante do modelo "Fulito". $\mathrm{O}$ número de tentativas com cada relação, porém, inviabilizava a redução gradual da apresentação das sequências de modelos.

$\mathrm{O}$ número de tentativas adotado no presente estudo foi definido a partir de uma série de pesquisas sobre aprendizagem de duas novas relações por crianças de diferentes idades e condições de desenvolvimento, e situava-se dentro da faixa da maioria dos estudos (Carr, 2003 - 10 tentativas por relação; Costa et al., 2013 - entre três e dez tentativas; Schafer \& Plunkett, 1998 - seis tentativas; Wilkinson et al., 2003 - três tentativas). Observou-se, porém, que possivelmente esse número foi pequeno para os bebês. Dos estudos citados, apenas o de Schafer e Plunkett foi realizado com bebês tão jovens (16 meses) e indicou que os bebês, de modo geral, aprenderam apenas uma das duas relações ensinadas (assim como no estudo de Liittschwager \& Markman, 1994 - em ambos os estudos, o ensino das relações era por pareamento auditivo-visual). Os demais estudos foram realizados com crianças mais velhas. É possível que o número de exposições, especialmente da segunda relação, que ocorreu após várias exposições sequenciais da primeira, aliado à ocorrência de muitos erros no desempenho da maioria das crianças, tenha dificultado o estabelecimento de um padrão estável de discriminação para as duas novas relações antes da realização dos testes, diferentemente do que foi encontrado em outros estudos com crianças mais velhas. Nesse sentido, parece importante se investigar não apenas a quantidade de exposições necessárias para que bebês abaixo dos 20 meses aprendam múltiplas relações palavra-objeto, mas também a distribuição dessas tentativas ao longo do tempo (Childers \& Tomasello, 2002; Riches, Tomasello, \& Conti-Ramsden, 2005), a eventual necessidade de associação de outros procedimentos ao ensino por exclusão, como o pareamento ostensivo (e.g., Sousa, Souza, \& Gil, 2013) e, principalmente, a forma de apresentação das tentativas de ensino.

O procedimento blocado, mesmo quando realizado de forma mais extensa e com a redução gradual do número de tentativas por bloco, pode não ser condição suficiente para gerar a aprendizagem de relações condicionais - arbitrárias ou de identidade (Saunders \& Spladlin, 1993). Apesar dele aparentemente favorecer a discriminação entre os estímulos modelo e os de comparação, talvez seja insuficiente para estabelecer adequadamente a condicionalidade entre as relações. A sequência de tentativas com um único modelo pode estabelecer um controle exclusivo pelo comparação, como em uma discriminação simples; a mudança do modelo, em outra sequência de tentativas, pode estabelecer uma mudança na seleção do estímulo de comparação como em uma reversão de discriminação simples, igualmente sendo insuficiente para produzir a condicionalidade entre modelos e comparações. De acordo com Saunders e Spradlin (1990; 1993), a introdução de uma resposta diferencial ao modelo no procedimento blocado pode ser importante para o estabelecimento da condicionalidade entre modelos e comparações e esta pode ser uma sugestão a ser investigada futuramente para procedimentos conduzidos com crianças com menos de 24 meses.

Uma última variável a ser considerada é a condução de sondas em extinção com bebês tão jovens. Carr (2003) chamou a atenção para a condução de tentativas de exclusão em extinção em procedimentos de ensino, descrevendo que a consequenciação dessas tentativas facilitava o desempenho de crianças com autismo. No presente estudo, as tentativas de ensino foram consequenciadas, mas em função da pouca estabilidade do desempenho da maioria dos participantes no ensino da segunda relação, é possível supor que a ausência de consequências diferenciais para erros e acertos nos testes tenha tido um efeito deletério sobre o desempenho dos participantes. Observa-se, por exemplo, que cinco dos participantes começaram o bloco de testes com acertos, e que P1, P3, P6 e P7 apresentaram uma sequência de pelo menos três acertos consecutivos (quatro, no caso de P6 e P7). No caso desses participantes, a ausência de consequências diferenciais pode ter surtido um efeito já discutido por Galvão, Calcagno e Sidman (1992): resultados negativos em testes de aprendizagem nestas condições podem mascarar os efeitos do ensino, pois o participante pode não entender a demanda do pesquisador ou, simplesmente, entender que o pesquisador não se importa com o seu desempenho (i.e., qualquer resposta é aceitável pelo pesquisador na tarefa).

Outra possibilidade é que a ausência de reforçamento em testes pode funcionar como punição para o desempenho do participante, competindo com o reforçamento diferencial da fase de ensino. Características próprias de bebês nessa faixa etária podem favorecer esses efeitos em tentativas de teste não reforçadas, o que deve ser melhor investigado em estudos futuros, uma vez que, como asseveram Oliveira e Gil (2008), bebês são uma população de especial interesse para o estudo de comportamento simbólico e é necessário que se estabeleçam condições experimentais que de fato favoreçam o estudo das variáveis críticas que atuam sobre o seu comportamento. Uma alteração possível em estudos futuros, nesse sentido, poderia ser a condução de blocos de teste não completamente em extinção, mas com a redução da probabilidade de reforçamento no bloco (e.g., 50\%), inserindo-se mais tentativas de linha de base que pudessem ser reforçadas. Deve-se ter cuidado, no entanto, para não estender o procedimento, o que pode afetar negativamente a motivação e $\mathrm{o}$ engajamento dos participantes, o que costuma ser uma dificuldade comum 
nesse tipo de procedimento com crianças pequenas (cf. Postalli et al., 2015)

Esse estudo teve algumas limitações que devem ser ponderadas na análise de seus resultados. A primeira delas refere-se ao fato de não ter sido realizada uma avaliação sistematizada do desenvolvimento dos bebês. A seleção dos bebês foi realizada com base no relato de pais e professores sobre o suposto desenvolvimento típico dos bebês e, portanto, não há garantias de que, de fato, os bebês apresentavam repertórios compatíveis com o de crianças de sua idade. Outra limitação é o fato do procedimento ter sido feito em uma única sessão, o que pode ter cansado os bebês, apesar da rapidez da sessão, o que pode ter impactado nos resultados obtidos.

Além disso, a instabilidade do desempenho dos bebês durante o ensino da segunda relação também pode ter tido um efeito negativo sobre os resultados dos testes e provavelmente isso ocorreu em função do tipo de procedimento de ensino planejado. Essas questões devem ser focalizadas em estudos futuros. Procedimentos alternativos para o ensino de múltiplas relações entre palavras e objetos para bebês devem incluir procedimentos mais curtos (conforme já indicado por Oliveira \& Gil, 2008), número maior de sessões e um procedimento que permita o estabelecimento de condicionalidade entre os estímulos modelo e comparação (alternância dos modelos em fase de ensino, antes da apresentação dos testes), bem como o planejamento de uma resposta diferencial ao modelo. Independente dessas limitações, porém, o presente estudo parece indicar aspectos importantes da aprendizagem de múltiplas relações por bebês e indica caminhos para futuras investigações sobre as variáveis implicadas na aprendizagem de vocabulário por crianças.

\section{REFERÊNCIAS}

Carey, S. \& Bartlett, E. (1978). Acquiring a single new word. Papers and Reports on Child Language Development, 15, 17-29.

Carr, D. (2003). Effects of exemplar training in exclusion responding on auditory-visual discrimination tasks with children with autism. Journal of Applied Behavior Analysis, 36, 507-524.

Childers, J. B., \& Tomasello, M. (2002). Two-year-olds learn novel nouns, verbs and conventional actions from massed or distributed exposures. Developmental Psychology, 38, 967-978.

Costa, A. R. A., Grisante, P. C., Domeniconi, C., de Rose, J. C., \& de Souza, D. G. (2013). Nomeação de estímulos novos a partir da seleção por exclusão. Paidéia, 23(55), 217-224.

Costa, A. R. A., McIlvane, W. J., Wilkinson, K. M., \& de Souza, D. G. (2001). Emergent word-object mapping by children: Further studies using the blank comparison technique. The Psychological Record, 51, 343-355.

de Souza, D. G., \& de Rose, J. C. (2006). Desenvolvendo programas individualizados para ensino de leitura e escrita. Acta Comportamentalia, 14, 77-98.

Danna, M. F., \& Matos, M. A. (2006). Aprendendo a observar. São Paulo: Edicon.
Dixon, L. S. (1977). The nature of control by spoken words over visual stimulus selection. Journal of the Experimental Analysis of Behavior, 27, 433-442.

Domeniconi, C., Costa, A. R. A., de Souza, D. G., \& de Rose, J. C. (2007). Responder por exclusão em crianças de 2 a 3 anos em uma situação de brincadeira. Psicologia: Reflexão e Crítica, 20, 342-350.

Dube, W. V., \& McIlvane, W. J. (1992). An analysis of generalized identity matching-to-sample test procedures. The Psychological Record, 42, 17-29.

Ferrari, C., de Rose, J. C., \& McIlvane, W. J. (1993). Exclusion vs. selection training of auditory-visual conditional relations. Journal of Experimental Child Psychology, 56, 49-63.

Galvão, O.F., Calcagno, S., \& Sidman, M. (1992). Testing for emergent performances in extinction. Experimental Analysis of Human Behavior Bulletin, 10(2), 18-20.

Golinkoff, R. M., Hirsh-Pasek, K., Bailey, L. M., \& Wenger, N. R. (1992). Young children and adults use lexical principles to learn new nouns. Developmental Psychology, 28, 99-108.

Greer, R. D., \& Du, L. (2015). Experience and the onset of the capability to learn names incidentally by exclusion. The Psychological Record, 65, 355-373.

Greer, R. D., \& Ross, D. E. (2008). Verbal behavior analysis: Inducing and expanding complex communication in children with severe language delays. Boston: Allyn \& Bacon.

Halberda, J. (2003). The development of a word-learning strategy. Cognition, 87, 23-34.

Horst, J. S., \& Samuelson, L.K. (2008). Fast mapping but poor retention by 24-month-old infants. Infancy, 13, 128-157.

Kucker, S. C., McMurray, B., \& Samuelson, L. K. (2015). Slowing down fast mapping: Redefining the dynamics of word learning. Child Development Perspectives, 9, 74-78.

Langsdorff, L. C., Domeniconi, C., Schmidt, A., de Souza, D. G., \& Gomes, C. G. (in press). Learning by exclusion in individuals with autism and Down syndrome. Psicologia: Reflexão e Crítica.

Langsdorff, L. C., Schmidt, A., \& Domeniconi, C. (2015). Aprendizagem de relações auditivo-visuais por meio de tentativas de exclusão. Interação em Psicologia, 19, 25-35.

Liittschwager, J. C., \& Markman, E. M. (1994). Sixteenand 24-month-olds' use of mutual exclusivity as a default assumption in second-label learning. Developmental Psychology, 30, 955-968.

Markman, E. M., Wasow, J. L., \& Hansen M. B. (2003). Use of the mutual exclusivity assumption by young word learners. Cognitive Psychology, 47, 241-275.

Mather, E., \& Plunkett, K., (2011). Mutual exclusivity and phonological novelty constrain word learning at 16 months. Journal of Child Language, 38, 933-950.

McIlvane, W. J., \& Stoddard, L. T. (1981). Acquisition of matching-to-sample performances in severe mental retardation: Learning by exclusion. Journal of Mental Deficiency Research, 25, 33-48. 
Oliveira, T. P., \& Gil, M. S. C. A. (2008). Condições experimentais facilitadoras para a aprendizagem de discriminação por bebês. Psicologia: Teoria $e$ Pesquisa, 24, 5-18.

Postalli, L. M. M., Canovas, D. S., \& de Souza, D. G. (2015). Simple discrimination and reversal learning sets in typically developing young children. The Psychological Record, 65, 411-423.

Riches, N. G., Tomasello, M., \& Conti-Ramsden, G. (2005). Verb learning in children with SLI: Frequency and spacing effects. Journal of Speech, Language, and Hearing Research, 48, 1397-1411.

Saunders, K. J., \& Spradlin, J. E. (1989). Conditional discrimination in mentally retarded adults: The effect of training the component simple discriminations. Journal of the Experimental Analysis of Behavior, 52, $1-12$.

Saunders, K. J., \& Spradlin, J. E. (1990). Conditional discrimination in mentally retarded adults: The development of generalized skills. Journal of the Experimental Analysis of Behavior, 54, 239-250.

Saunders, K. J., \& Spradlin, J. E. (1993). Conditional discrimination in mentally retarded subjects: Programming acquisition and learning set. Journal of the Experimental Analysis of Behavior, 60, 571-585.

Schafer, G., \& Plunkett, K. (1998). Rapid word learning by fifteen-month-olds under tightly controlled conditions. Child Development, 69, 309-320.
Schmidt, A., Franco, M. G. O., Lotério, L. S., \& Gomes, G. F. (2016). Learning name-object relations after a single exclusion trial in 18- to 48-month-old children. The Psychological Record, 66, 53-63.

Schwartz, R., \& Terrell, B. (1983). The role of input frequency in lexical acquisition. Journal of Child Language, 10, 57-66.

Sousa, N. M., Souza, C. B. A., \& Gil, M. S. C. A. (2013). Aprendizagem rápida de comportamento de ouvinte por um bebê. Interação em Psicologia, 17(1), 67-78.

Wilkinson, K. M., \& Green, G. (1998). Implications of fast mapping for vocabulary expansion in individuals with mental retardation. Augmentative and Alternative Communication, 14(3), 162-170.

Wilkinson, K. M., \& McIlvane, W. J. (1997). Blank comparison analysis of emergent symbolic mapping by young children. Journal of Experimental Child Psychology, 67(2), 115-13.

Wilkinson, K. M., Rosenquist, C., \& McIlvane, W. J. (2009). Exclusion learning and emergent symbolic category formation in individuals with severe language impairments and intellectual disabilities. The Psychological Record, 59, 187-206

Wilkinson, K. M., Ross, E., \& Diamond, A. (2003). Fast mapping of multiple words: Insights into when "the information provided" does and does not equal "the information perceived". Applied Developmental Psychology, 24, 739-762. 